\title{
Importance of Inhaler-Device Satisfaction in Asthma Treatment: Real-World Observations of Physician-Observed Compliance and Clinical/Patient-Reported Outcomes
}

\author{
M. Small · P. Anderson · A. Vickers $\cdot$ S. Kay $\cdot$ S. Fermer
}

Received: December 22, 2010 / Published online: February 10, 2011

(C) The Author(s) 2011. This article is published with open access at Springerlink.com

\section{ABSTRACT}

Introduction: It is hypothesized that health and patient-reported outcomes in asthma are positively influenced by the level of patient satisfaction with their inhaler device. This paper uses data from a real-world observational study to investigate the extent of the relationship between inhaler satisfaction and patient compliance, and the influence this has on health and patient-reported outcomes. Methods: Data were drawn from the Adelphi Respiratory Disease Specific Programme ${ }^{\circledR}$ (Adelphi, Macclesfield, UK), a cross-sectional study of consulting patients in five European countries undertaken between June and September 2009. A range of clinical and patient-reported outcomes were observed allowing analysis of these and their relationship with patient-reported inhaler satisfaction and patient compliance. Results: The analysis demonstrates that for the majority of patients the higher the level of satisfaction that the

M. Small · P. Anderson $(\bowtie) \cdot$ A. Vickers · S. Kay • S. Fermer

Adelphi Real World, Adelphi Mill, Grimshaw Lane, Macclesfield, SK10 5JB, UK.

Email: peter.anderson@adelphigroup.com patient reports for their device the more likely the patient is observed to be compliant and to experience better outcomes including quality of life (as measured by EuroQol 5 Dimensions [EQ5D] utility score, $P<0.001)$, fewer exacerbations $(P<0.001)$, fewer hospital visits $(P=0.011)$, fewer healthcare visits $(P=0.001)$, fewer primary care physician visits $(P=0.001)$, and fewer sleep disturbances $(P<0.001)$. Conclusion: The level of patient satisfaction with their inhaler device is observed to have a positive influence on the treatment goals for asthma through its association with improved compliance.

Keywords: asthma; compliance; device; health outcomes; inhaler; patient-reported outcomes

\section{INTRODUCTION}

Asthma is a common inflammatory condition of the upper respiratory tract estimated to affect approximately 300 million people worldwide ${ }^{1}$ and with prevalence increasing globally by $50 \%$ every decade. $^{2}$

Recent studies in Europe, ${ }^{3}$ the United States, ${ }^{4}$ Japan, ${ }^{5}$ and the Asia-Pacific region ${ }^{6}$ indicate that asthma is underdiagnosed and undertreated and that there is considerable 
room for improvement in the management of the condition.

Asthma is commonly treated with bronchodilator and anti-inflammatory drugs, in the majority of cases delivered by inhaler as advocated by current treatment guidelines. ${ }^{7}$ Today there exists a wide choice of inhalers and improvements are constantly being developed to optimize drug delivery and ease of use. Improvement in the management of the condition is therefore reliant not only on the medications themselves but also on their effective delivery. ${ }^{8}$ Poor inhaler technique is common and associated with noncompliance. ${ }^{9}$ The investigators selected the degree of satisfaction with the inhaler as a reliable indication of a patient's ability to successfully use their inhaler and thus potentially improve their compliance. In this context, satisfaction is defined as the extent to which the inhaler fulfills the desires, expectations, and requirements of the patient in terms of the patient's expectation of the features of the inhaler. The investigators went on to study the association between satisfaction with the inhaler device and compliance.

This paper focuses on the behavioral and attitudinal evidence collected by one observational and health outcomes research-based organization under the label of Disease Specific Programmes (DSPs ${ }^{\circledR}$; Adelphi, Macclesfield, UK). The DSPs are large, multinational, observational studies of clinical practice, designed to capture a cross-section of real-world data. These are an established method for investigating current treatment practices across a wide range of disease areas. ${ }^{10}$ The Respiratory DSP accurately reflects the current symptom prevalence and severity among the consulting population and associated treatment practices of respiratory conditions including asthma. ${ }^{11}$

The present work investigates the extent of the relationship between inhaler satisfaction and patient compliance and the influence this has on health and patient-reported outcomes.

\section{METHODS}

\section{Study Design}

The Respiratory DSP was conducted between June and September 2009, including specialists and primary care physicians and their patients in France, Germany, Italy, Spain, and the UK. Physicians completed a patient record form (PRF) for six consecutive consulting adult asthma patients, and the same patients were invited to fill out a self-completion form (PSC). The full methodology for this survey has been outlined previously. ${ }^{12}$

Physicians were identified by the local DSP fieldwork teams from public lists of healthcare professionals according to predefined selection criteria to ensure that the sample was representative of asthma management in each participating country. Physicians were checked for their eligibility to participate in the DSP in terms of specialty, whether they are personally responsible for treatment decisions, and how many patients they see in a typical week (in total and with asthma in order to avoid physicians with an abnormal workload). Candidate respondents who met the predefined eligibility criteria were subsequently invited to participate in the study. To avoid potential selection bias due to variable population densities in different geographical regions in a given country, an appropriately larger sample of physicians was identified in densely populated areas than in more sparsely populated areas. As the methodology requires the next six presenting asthma patients for each physician, the DSP sample is representative of the consulting population. Patients were invited by the physician to complete the PSC independently and return it in a sealed envelope. 
At no stage does the physician see or influence the responses made by the patient.

All responses were voluntary and anonymous to preserve patient confidentiality and to avoid bias at the data collection and analysis phases. The study adheres to version 6.3 of the European Pharmaceutical Market Research Association (EphMRA) code of conduct governing market research, ${ }^{13}$ including written informed consent of all patients for anonymous and aggregated reporting of research findings based on the questionnaires employed. Matching the physician and patient responses via patient/ physician study numbers allows the PSC data to be linked with comparable data recorded on the physician-completed PRF to highlight any areas of disparity and/or agreement.

\section{Patient Inhaler Satisfaction}

Patients were asked to rate their satisfaction across 13 attributes specific to their asthma inhaler device on a seven-point Likert scale, as well as their overall satisfaction with the device. The list of attributes (see Table 1) was created by Adelphi for the Respiratory DSP in consultation with physicians.

\section{Compliance}

The cross-sectional nature of the DSP methodology does not permit the observation of actual compliance. For the purposes of this paper, therefore, the term compliance is defined as the extent to which the patients are perceived to follow their physician's prescribing instructions and advice. Physicians were asked to rate separately patients' compliance with regards to frequency of administrations per day and inhaler device usage. Both were measured using five-point Likert scales, where $1=$ not at all compliant and 5=fully compliant.
Table 1. List of 13 attributes associated with inhaler usage.

No. Attribute

1. It is built to last and will not break easily

2. It is easy to hold and carry

3. The instructions are simple and easy to follow

4. No need for me to put the drug into the inhaler before I use it

5. I do not need to breathe in at the same time as I press my inhaler

6. I do not need to breathe in hard to inhale my medication

7. I get the same amount of medication delivered to my lungs each time

8. It tells me when my dose of medication has been inhaled correctly

9. It tells me how many doses of my medicine I have left

10. It does not need to be cleaned

11. The inhaler locks when it is empty so I cannot use it anymore

12. I can throw the inhaler away and get a new one with each prescription

13. I keep the inhaler and refill it myself with each prescription.

Use of a Likert scale can be considered a suitable point in time method of measuring compliance in that it demonstrates enough sensitivity to differentiate any patient's compliance relative to others. ${ }^{14}$

\section{Health and Patient-Reported Outcomes}

The following health and patient-reported outcomes were analyzed against the compliance rating provided by physicians: quality of life (as measured by the EuroQol 5 Dimensions [EQ-5D] utility score), exacerbations, hospital visits, healthcare visits, and sleep disturbances (as measured 
by the Jenkins Sleep Scale $\left.{ }^{15}\right)$. The EQ-5D and Jenkins Sleep Scale are recognized instruments for measuring patient reported outcomes. For exacerbations, hospital visits, and healthcare visits, we used standard questions taken from the DSP methodology.

\section{Statistical Methods}

Ordinary least squares regression was used to relate compliance to overall satisfaction with inhaler, severity, gender, age, ethnicity, and number of maintenance drugs (log transformed). A generalized additive model ${ }^{16}$ was used to investigate further the relationship for compliance to overall satisfaction with inhaler, without making any assumptions for the shape of the association. Sequential regression with average over orderings and bootstrap confidence intervals ${ }^{17}$ was used to investigate the relationship with overall satisfaction and inhaler characteristics. This method is appropriate where the independent variables are highly correlated with each other. ${ }^{17}$ The associations of the health outcome data with compliance with inhaler were modeled using generalized additive models, which again made no assumption about the shape of the relationship.

\section{RESULTS}

A total of 582 physicians participated in the study (see Table 2) providing a total of 2135 patient records (see Table 3 ).

A total of 1400 patients provided PSCs and these were matched to the corresponding physician-completed PRFs. Of these 1400 patients, 1345 answered the inhaler attribute satisfaction section of the PSC (France 311; Germany 328; Italy 316; Spain 295; UK 95).

In order to generate one compliance rating score for each patient, the two Likert scales measuring frequency and inhaler usage were tested for their degree of correlation and showed a Cronbach's alpha of 0.924. Since the widely accepted social science cut-off level is that alpha should be 0.7 or higher for a set of items to be considered a scale, ${ }^{18}$ the overall mean score was taken as the compliance rating for each patient (see Figure 1). Therefore for the majority of patients, the physician gave the patient the same score so the mean score is a whole number between 1 and 5. For those patients whose physician gave them a different score on the two scales, their overall rating is the mean of the two scores, for example 4 and $5=4.5$.

In the case of physician-perceived reporting used for this study, 63\% of physicians gave a

Table 2. Study physicians: distribution and specialty.

\begin{tabular}{lcccccc}
\hline Physicians & France & Germany & Italy & Spain & UK & Total \\
\hline Primary care physicians & 50 & 50 & 50 & 50 & 52 & 252 \\
Pulmonologists & 50 & 50 & 50 & 50 & 50 & 250 \\
Allergologists & 20 & 20 & 20 & 20 & - & 80 \\
\hline Total & 120 & 120 & 120 & 120 & 102 & 582 \\
\hline
\end{tabular}

Table 3. Study patients: distribution by country and specialty of physician who completed the patient record.

\begin{tabular}{lcccccc}
\hline Physicians & France & Germany & Italy & Spain & UK & Total \\
\hline Primary care physicians & 196 & 264 & 220 & 195 & 111 & 986 \\
Specialists & 217 & 384 & 294 & 210 & 44 & 1149 \\
\hline Total & 413 & 648 & 514 & 405 & 155 & 2135 \\
\hline
\end{tabular}


different compliance rating for at least two of their six patient records provided. This provides evidence that physicians were rating patient compliance relative to their other patients.

Figure 1 shows that the mean physicianperceived compliance rating follows a highly skewed distribution with $49 \%$ of patients $(n=1051)$ given the fully compliant rating of 5 on both scales and the remaining $51 \%$ of patients $(n=1084)$ considered less than fully compliant to a greater or lesser degree.

Figure 2 shows the highest mean scores for patient-reported satisfaction with their inhaler related to those attributes which reflect convenience, durability, and simplicity: easy to hold and carry, no need to put the drug into the inhaler before use, built to last/will not break easily, and simple and easy to follow instructions. Sequential regression with average over orderings and bootstrap 95\% confidence intervals was used to present the list of inhaler attributes according to which is most predictive of overall inhaler satisfaction (or dissatisfaction). As can be seen in Figure 3, the top five drivers of inhaler satisfaction were: built to last/will not break easily, easy to hold and carry, instructions are simple and easy to follow, I get the same amount of medication delivered to my lungs

Figure 1. Distribution of physician-perceived patient compliance (mean of two 1-5 scales). Base: all adult asthma patients. ${ }^{*}$ A compliance rating of 5 on one scale and 4 on the other gives a mean rating of 4.5 for that particular patient.

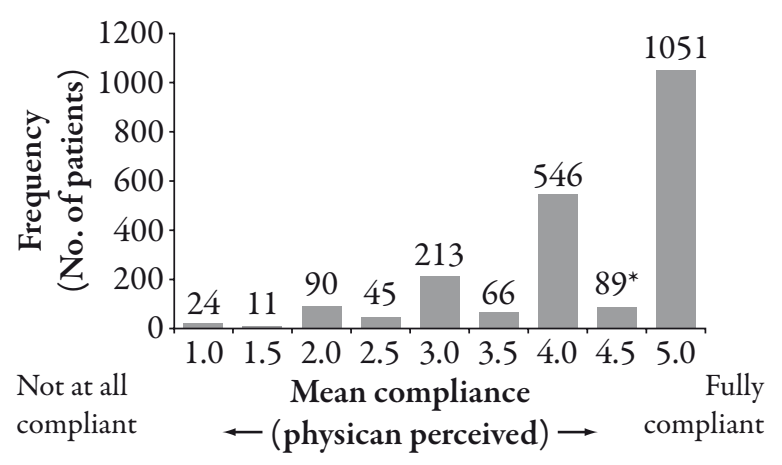

each time, and no need to put the drug into the inhaler before use.

Four of these attributes (1, 2, 3, and 5) are related to convenience for the patient and together these account for $68 \%$ of the explained variation contributing to overall satisfaction.

Figure 4 shows the degree of the relationship between patient-reported overall satisfaction with their inhaler device and compliance while considering the influence of a number of confounding variables: age, gender, ethnicity, number of maintenance drugs, and physicianperceived severity.

There was a significant relationship $(P<0.001)$ between the level of compliance and patient-reported satisfaction with their inhaler device. For the total patient sample, the confounding measures were all found to be insignificant and therefore have no relationship with compliance.

Figure 5 shows that a higher degree of inhaler satisfaction is associated with a significantly better level of physician-perceived compliance $(P<0.001)$.

\section{Observation of the Relationships between Device Satisfaction, Compliance, and Health and Patient-Reported Outcomes}

In this study, the majority of patients (92\%) support the hypothesis that health and patientreported outcomes in asthma are positively influenced by the level of patient satisfaction with their inhaler device. For these patients, improved quality of life, reduced number of exacerbations, reduced visits to primary care practitioner and other healthcare professionals (asthma nurses, for example), and reduction in sleep disturbance were shown to be associated with the level of satisfaction with their device and consequently physician-reported level of compliance. For $100 \%$ of patients, a reduction 
Figure 2. Patient satisfaction with specific inhaler attributes. Error bars show bootstrap $95 \%$ confidence intervals.

$$
\begin{array}{r}
\text { It is easy to hold and carry } \\
\text { No need for me to put the drug into the inhaler before I use it } \\
\text { It is built to last and will not break easily } \\
\text { The instructions are simple and easy to follow }
\end{array}
$$

I can throw the inhaler away and get a new one with each prescription

I get the same amount of medication delivered to my lungs each time

It does not need to be cleaned

I do not need to breathe in at the same time as I press my inhaler It tells me how many doses of my medicine I have left I do not need to breathe in hard to inhale my medication

The inhaler locks when it is empty so I cannot use it any more It tells me when my dose of medication has been inhaled correctly

I keep the inhaler and refill it myself with each prescription

Not at all satisfied

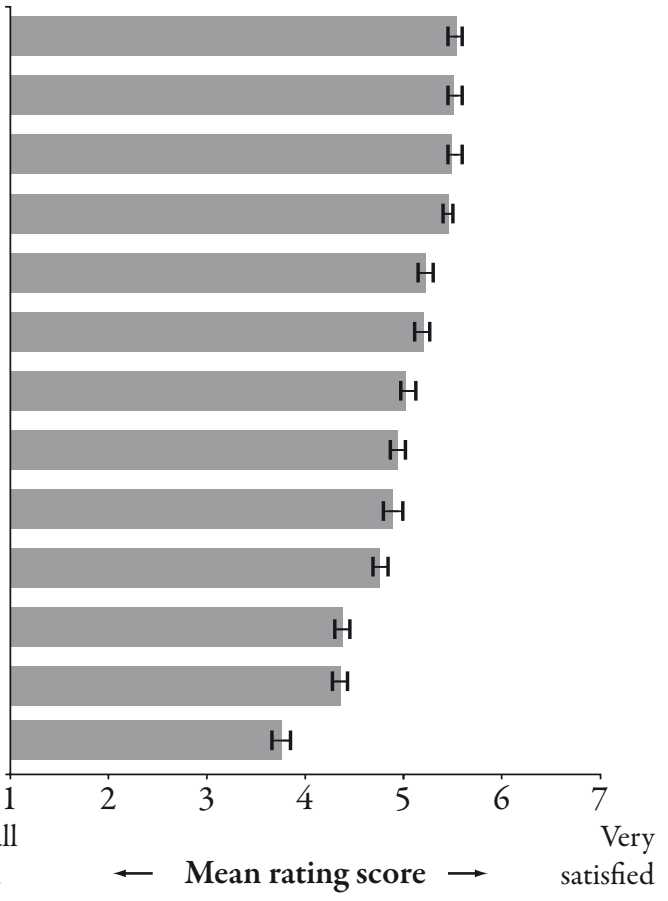

Figure 3. Influence of patient-reported satisfaction with specific inhaler attributes on overall satisfaction. ${ }^{*}$ Percentage of $\mathrm{R}^{2}$ (total $\left.\mathrm{R}^{2}=0.613\right)$.

It is built to last and will not break easily It is easy to hold and carry

The instructions are simple and easy to follow

I get the same amount of medication delivered to my lungs each time

No need for me to put the drug into the inhaler before I use it

I do not need to breathe in at the same time as I press my inhaler

I do not need to breathe in hard to inhale my medication

I can throw the inhaler away and get a new one with each prescription It tells me how many doses of my medicine I have left It does not need to be cleaned

It tells me when my dose of medication has been inhaled correctly

The inhaler locks when it is empty so I cannot use it any more

I keep the inhaler and refill it myself with each prescription

$$
\text { ( }
$$

Not at all satisfied

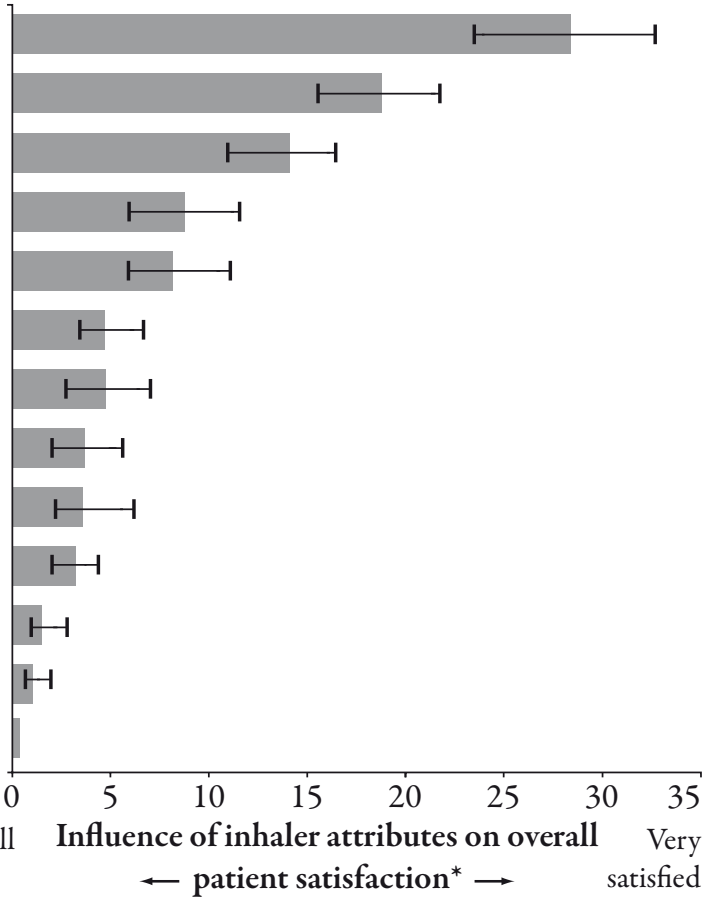


Figure 4. Degree of association of compliance with overall inhaler satisfaction and selected patient characteristics.

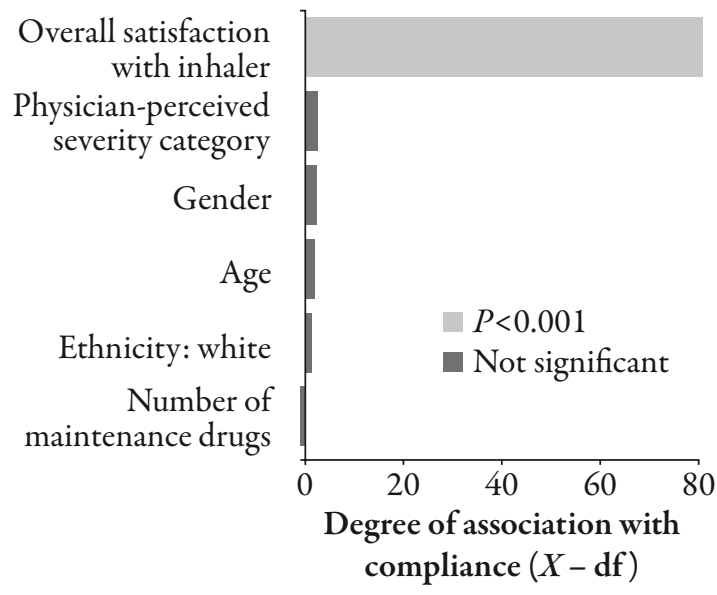

Figure 5. Relationship of compliance rating and overall inhaler satisfaction. $\mathrm{R}^{2}=0.060 . P<0.001$.

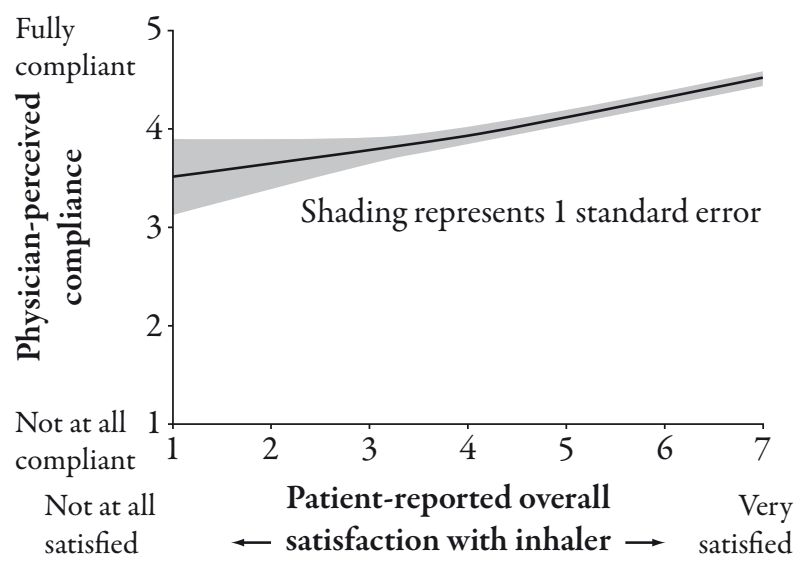

in the number of hospital visits was associated with the level of satisfaction with their device and associated physician-reported level of compliance.

Figures 6 to 11 illustrate the relationship between physician-perceived compliance and EQ-5D utility score, number of exacerbations in the last 12 months, hospitalizations as a result of an exacerbation, primary care physician visits, combined healthcare professional visits, and sleep disturbance. In each instance it was observed that a higher physician-perceived compliance rating was significantly associated
Figure 6. Higher physician-perceived compliance rating is associated with a better EuroQol 5 Dimensions (EQ-5D) utility score $(P<0.001)$. Shading represents $95 \%$ confidence interval. Base: all adult asthma patients. QoL $=$ quality of life.

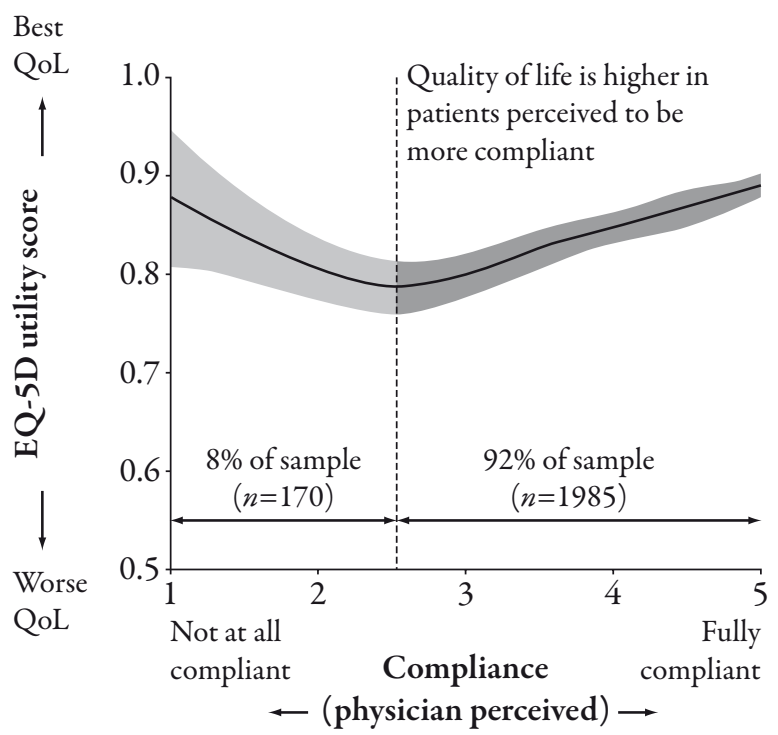

Figure 7. Higher physician-perceived compliance rating is associated with experiencing a lower number of exacerbations in the last 12 months as reported by their physician $(P<0.001)$. Shading represents $95 \%$ confidence interval. Base: all adult asthma patients.

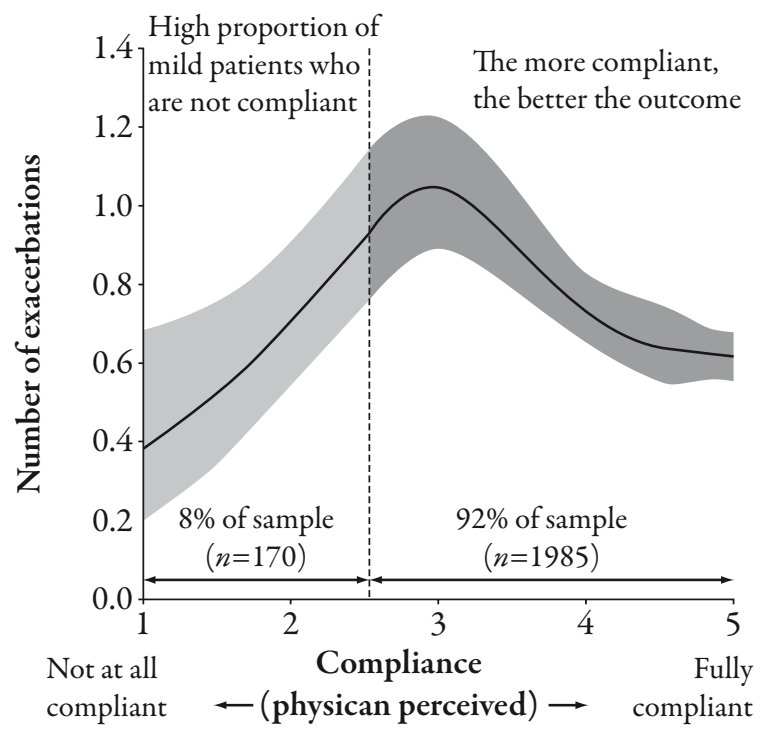


Figure 8. Higher physician-perceived compliance rating is associated with fewer attacks severe enough to require a visit to their primary care physician in the last 12 months as reported by the patient $(P=0.001)$. Shading represents $95 \%$ confidence interval. Base: all adult asthma patients.

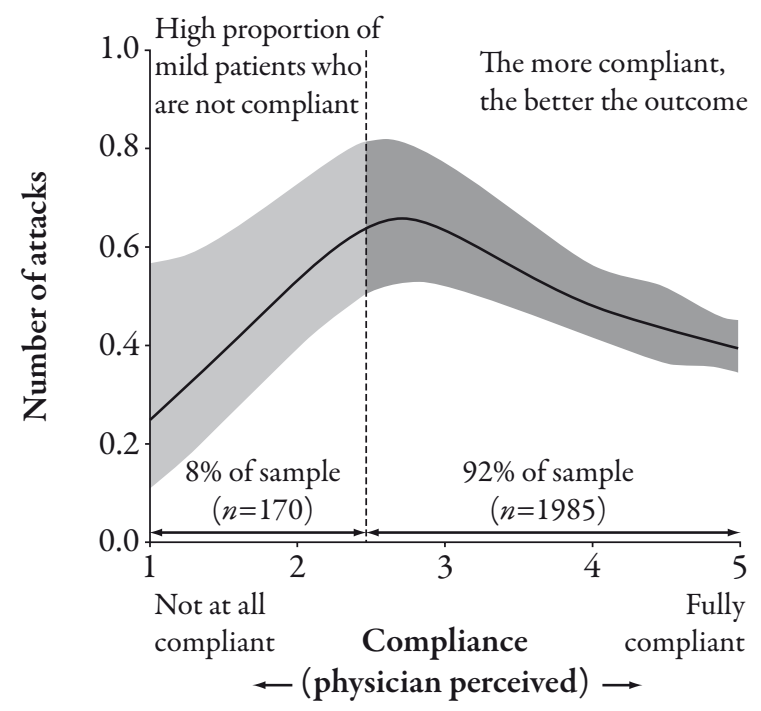

Figure 9. Higher physician-perceived compliance rating is associated with making fewer visits to a healthcare professional in the last 12 months as reported by the patient $(P=0.001)$. Shading represents $95 \%$ confidence interval. Base: all adult asthma patients.

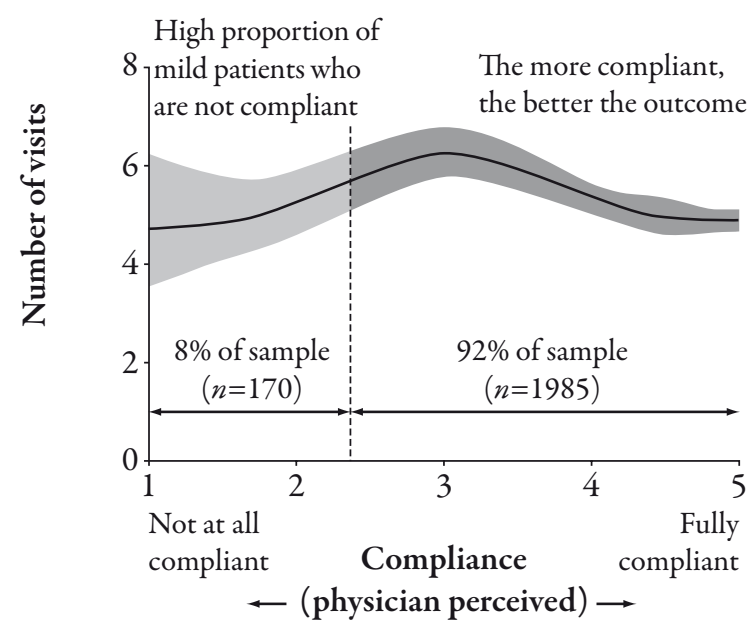

Figure 10. Higher physician-perceived compliance rating is associated with a lower number of hospital visits as a result of an exacerbation in the last 12 months as reported by the physician $(P=0.011)$. Shading represents $95 \%$ confidence interval. Base: all adult asthma patients.

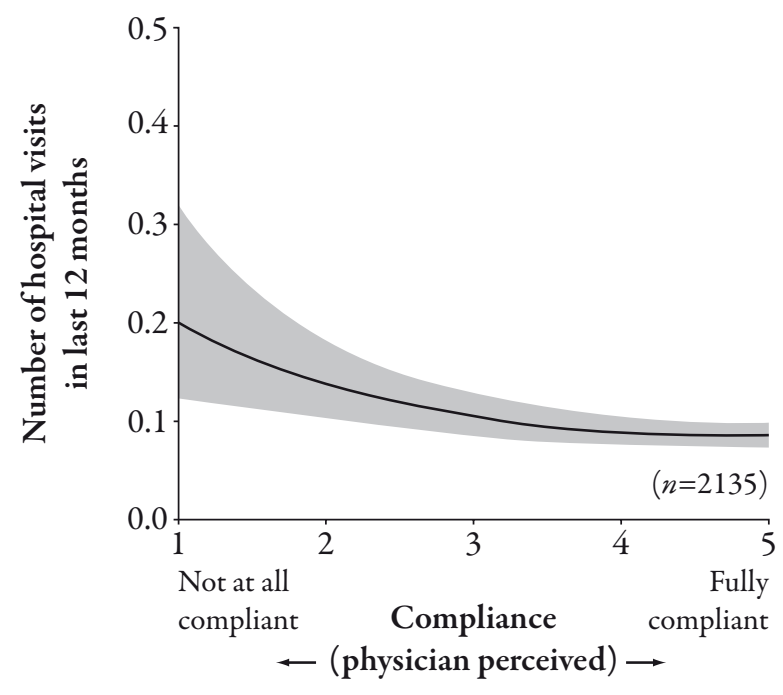

Figure 11. Higher physician-perceived compliance rating is associated with reporting fewer sleep disturbances $(P<0.001)$. Shading represents $95 \%$ confidence interval. Base: all adult asthma patients.

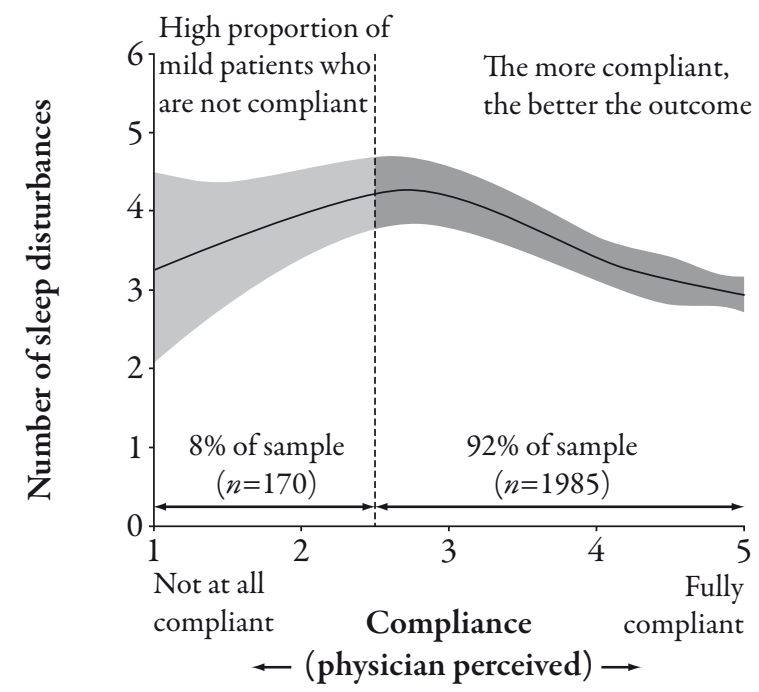


with a better outcome for the patient in terms of a higher utility score and fewer exacerbations, hospitalizations, healthcare professional visits, and sleep disturbances. This was shown to be consistent for the same $92 \%$ of the sample for all outcome variables with the exception of hospitalizations due to an exacerbation when it was applicable for all patients. For $8 \%$ of patients this association was not observed.

\section{DISCUSSION}

There is a clear need for accurate understanding of asthma management and the impact that realworld clinical practice by physicians has on the improved health and patient-reported outcomes of asthma patients. Behavioral and attitudinal evidence is one data source that can inform our understanding of asthma management. ${ }^{10}$

In asthma management, the inhaler device has an important and predominant place in the treatment armamentarium. The Adelphi Respiratory DSP collects behavioral and attitudinal evidence from asthma patients about their preferences and perceptions for the inhaler they are using. The DSP also collects data on the physician-perceived compliance of the same patient. Our results on the level of compliance and patient-reported satisfaction with their inhaler device complement previous research suggesting that adherence cannot be explained by certain patient characteristics such as patient age and gender or race. ${ }^{8}$

We collected objective observational data from both patients and physicians that allowed us to assess health and reported outcomes. In this study we developed a technique to allow us to study the relationship between these three areas, as it was felt that the behavior and attitude of patients towards their inhaler device may influence their health and patientreported outcomes.
Real-world data are valuable tools to develop our understanding of the behavior and attitude of patients in the context of their treatment. This understanding can assist us in designing improved treatment management strategies, which in turn may improve the outcome of the asthma patient; a widely accepted unmet need. ${ }^{3-6}$ We accept that there are inevitable limitations when collecting real-world data, specifically the selection and diagnosis of patients, as in the absence of formal randomization this is contingent on the integrity of the participating physicians rather than formal source verification procedures. Moreover, diagnosis and assessment of outcome is based on the judgment and diagnostic skills of the respondent physician. ${ }^{10}$ Although the DSP can be used to identify association, a further limitation is that being cross-sectional it cannot be used to demonstrate cause and effect. Additionally, the association between satisfaction and health and patient-reported outcomes may be explained by other factors not explored in this study such as the relationship between patient and physician and the disposition of the individual patient. Despite these limitations, the results provide helpful and objective evidence of the importance in terms of health and patient-reported outcomes of patient satisfaction with their device.

The physician's perception or reporting of compliance in this study was captured via a Likert scale, and represents one of a number of approaches that can be employed to measure the degree of patient compliance. Other approaches include self-reporting, pill counts, inhaler weighing, pharmacy records, and drug level monitoring. These have been evaluated in detail elsewhere and each has been shown to have its own strengths and weaknesses. ${ }^{19,20}$

In the majority of patients (92\%), this study observed an association on a number of health and patient-reported outcomes with the level of satisfaction with device and with physician- 
reported compliance. Further analysis of the small proportion $(8 \%)$ of patients who did not show this association, indicates that these patients are classified by their physician as being very mild and are perceived by the physician as being noncompliant. For these patients the analysis shows that poor compliance is associated with better outcomes. This observation is consistent with observations made previously whereby adherence, and by implication compliance, is influenced by the patients' perception of their disease, and a lack of symptoms is interpreted by patients as a disease that can be treated episodically. ${ }^{20}$

The importance of patient involvement in their disease and the desirability of a partnership between patient and physician is widely accepted. ${ }^{12}$ Our findings suggest that, for the majority of asthma patients, satisfaction with their device directly associates with their compliance and this correlates with improved outcome on a number of relevant clinical and patient-reported measures. Therefore increased patient involvement in the choice of device, and thus development of concordance as well as wider recognition of the important features that device manufacturers should consider when developing or enhancing inhaler devices, might be expected to result in an improved outcome for the asthma patient.

In our study we measured physician-reported perception of compliance. We believe a future study should investigate the relationship between treatment adherence and device satisfaction, and should collect treatment adherence data from the patient using a robust instrument such as the Beliefs in Medicine Questionnaire. ${ }^{8}$ We believe that randomized controlled studies assessing the potential of new devices to improve asthma treatment should also measure satisfaction with device, alongside measurement of lung deposition.

\section{CONCLUSION}

Overall patient satisfaction with their inhaler device is an important factor in encouraging patient compliance. If physicians made particular effort to select an inhaler that satisfies the patient, including attention to features associated with convenience, patient compliance with their asthma treatment would be expected to improve. This study further demonstrates the relationship of good compliance with better outcomes of the patient and points to a need for physicians to understand the central role that patient satisfaction with inhaler device plays in achieving better patient compliance.

\section{ACKNOWLEDGMENTS}

This study was supported by AstraZeneca. All listed authors meet the criteria for authorship set forth by the International Committee for Medical Journal Editors. PA is the guarantor for this article, and takes responsibility for the integrity of the work as a whole.

Open Access. This article is distributed under the terms of the Creative Commons Attribution Noncommercial License which permits any noncommercial use, distribution, and reproduction in any medium, provided the original author and source are credited.

\section{REFERENCES}

1. Braman S. The global burden of asthma. Chest. 2006;130:4S-12S.

2. Masoli M, Fabian D, Holt S, et al. Global Initiative for Asthma (GINA) program: the global burden of asthma: executive summary of the GINA Dissemination Committee Report. Allergy. 2004;59: 469-478.

3. Rabe KF, Vermeire PA, Soriano JB, et al. Clinical management of asthma in 1999: the Asthma 
Insight and Reality in Europe (AIRE) study. Eur Resp J. 2000;16:802-807.

4. GlaxoSmithKline. Asthma in America: a landmark survey. Available at: www.asthmainamerica.com. Accessed March 25, 2010.

5. Adachi M, Morikawa A, Ishihara K. Asthma insights and reality in Japan (AIRJ). Arerugi. 2002;51: $411-420$

6. Lai CK, De Guia TS, Kim YY, et al. Asthma control in the Asia-Pacific region: the Asthma Insights and Reality in Asia-Pacific Study. J Allergy Clin Immunol. 2003;111:263-268.

7. Global Initiative for Asthma. GINA report, Global Strategy for Asthma Management and Prevention 2009. Available at: www.ginasthma.org. Accessed: November 24, 2010.

8. Horne R. Compliance, adherence and concordance. Implications for asthma treatment. Chest. 2006;130(Suppl. 1):65S-72S.

9. Ward M, Reynolds C. Patient empowerment: the key to compliance in asthma. Nursing Times. 2000;96:8.

10. Anderson P, Benford M, Harris N, et al. Realworld physician and patient behaviour across countries: disease-specific programmes - a means to understand. Curr Med Res Opin. 2008;24: 3063-3072.

11. Higgins V, Kay S, Small M. Physician and patient survey of allergic rhinitis: methodology. Allergy. 2007;62:6-8.
12. Small M, Vickers A, Anderson P, Kay S. The patientphysician partnership in asthma: real-world observations associated with clinical and patientreported outcomes. Adv Ther. 2010;27:511-599.

13. European Pharmaceutical Market Research Association Code of Conduct for International Healthcare Market Research. Available at: www. ephmra.org/professional-standards.aspx. Accessed September 10, 2010.

14. Ley P, Llewellyn S. Improving patients' understanding, recall, satisfaction and compliance. In: Broome, A, Llewellyn S, eds. Health Psychology: Process and Applications. 2nd edition. London: Chapman and Hall; 1995:75-98.

15. Jenkins CD, Stanton BA, Niemcryk SJ, Rose RM. A scale for the estimation of sleep problems in clinical research. J Clin Epidemiol. 1988;41:313-321.

16. Wood S. Generalized Additive Models: an Introduction with R. Boca Raton, FL: Chapman and Hall/CRC; 2006.

17. Grömping U. Relative importance for linear regression in R: the package relaimpo. J Stat Soft. 2006;17:1-27.

18. Nunnally J, Bernstein I. Psychometric Theory. 3rd Edition. New York: McGraw Hill; 1994.

19. Cochrane GM. Compliance in asthma: a European perspective. Eur Respir J. 1995;5:116-119.

20. Bourbeau J, Bartlett S J. Patient adherence in COPD. Thorax. 2008;63:831-838. 